\title{
Results of Magnetic Field Measurements Performed with the 6-m Telescope. IV. Observations in 2010
}

\author{
I. I. Romanyuk, ${ }^{1,}{ }^{*} \quad$ E. A. Semenko, ${ }^{1}$ D. O. Kudryavtsev, ${ }^{1}$ A. V. Moiseeva, ${ }^{1}$ and I. A. Yakunin ${ }^{1}$ \\ ${ }^{1}$ Special Astrophysical Observatory, Russian Academy of Sciences, Nizhnii Arkhyz, 369167 Russia
}

\begin{abstract}
We present the results of measurements of magnetic fields, radial velocities and rotation velocities for 92 objects, mainly main-sequence chemically peculiar stars. Observations were performed at the 6-m BTA telescope using Main Stellar Spectrograph with a Zeeman analyzer. In 2010, twelve new magnetic stars were discovered: HD 17330, HD 29762, HD 49884, HD 54824, HD 89069, HD 96003, HD 113894, HD 118054, HD 135679, HD 138633, HD 138777, $\mathrm{BD}+53.1183$. The presence of a field is suspected in HD 16705, HD 35379 and HD 35881 . Observations of standard stars without a magnetic field confirm the absence of systematic errors which can introduce distortions into the measurements of longitudinal field. The paper gives comments on the results of investigation of each star.
\end{abstract}

\section{INTRODUCTION}

We continue an ensemble of papers on the study of magnetic stars at the 6-m telescope, initiated in Romanyuk et al. [1-3] by a publication of complete reports on the measurement of magnetic fields from the 2007-2009 observational data. This paper presents the results of measurements of magnetic fields in chemically peculiar stars, observed with the Main Stellar Spectrograph (MSS) of the 6-m BTA telescope of the Special Astrophysical Observatory of the Russian Academy of Sciences (SAO RAS) in 2010. The scientific substantiation of studies of our series is given in previous publications. Equipment, methods of observations and data reduction in general terms have not been changed in comparison with previous years. However, some

\footnotetext{
Electronic address: roman@sao.ru
}

changes did take place. Firstly, since March 2010 a new large-format CCD sized $4600 \times 2000$ pixels is used as a detector at the MSS. Secondly, the data reduction technique, described in [1-3] has been generally saved, however, a part of the field measurements was also performed using the regression method [4].

\section{OBSERVATIONS AND REDUCTION METHOD}

The material that served as the basis for this study was obtained at the 6-m telescope in 2010 during 21 nights of observations within four main programs: Magnetic Fields of Massive Stars (principal applicant: I. I. Romanyuk, SAO RAS); New Magnetic Stars (principal applicant: D. O. Kudryavtsev, SAO RAS); Selected Magnetic Stars (principal applicant: E. A. Semenko, SAO RAS); Geometry of Magnetic Fields 
in CP Stars (principal applicant: G. Wade, mately the same results. However, there are sigCanada). One hundred eighty-four pairs of circu- nificant differences for fast rotators. As a rule, larly polarized spectra were obtained for 92 stars. the longitudinal fields, obtained by the regresThe list of objects is significantly different from sion method, turn out to be substantially smaller the similar set of previous years.

than those measured by the classical method. In

A part of observations (in January and February 2010) was conducted, just like in 2009, using a CCD sized $2000 \times 2000$ pixels. Since March 25, a new CCD array sized $4600 \times 2000$ pixels has been used for the observations. An introduction of a new panoramic CCD allowed to expand the simultaneously registered spectral range up to $550 \AA$, which significantly improved the possibilities for observing magnetic fields of stars. In most cases, we worked in the spectral range from $4450 \AA$ to $5000 \AA$.

We have earlier repeatedly described the preliminary reduction procedure of the spectral data (image processing, extraction of spectra, wavelength calibration, etc.) and measurement of magnetic fields by the modified Babcock method, for example, in [1-3]. In addition, new software that allows determining the longitudinal magnetic field of stars by the regression method proposed by Bagnulo et al. [4] was applied. This method proved to be very useful in the studies of fast rotators with complex line profiles for which the classical measurement procedure led to a large scattering of results.

When measuring the magnetic fields of stars with narrow lines, both methods give approxiaddition to the longitudinal magnetic fields $B_{e}$, we determined radial velocities $V_{r}$ and projections of rotation velocities onto the line of sight $v_{e} \sin i$ for each star.

\section{RESULTS OF MEASUREMENTS}

The results of magnetic field measurements are presented in the summary Table. The columns of the table contain data on the names of stars in the order of increasing numbers in the HD and BD catalogs, on the Julian date of observations, the longitudinal field $B_{e}$, the radial velocity $V_{r}$, and rotation velocity $v_{e} \sin i$ with measurement errors $\sigma$, and the $S / N$ ratio of the resulting spectrum. The longitudinal field, measured by the standard Babcock method is marked with the letter "z", by the regression method-by the letter "r", measurements of the magnetic field by the core of the hydrogen $\mathrm{H} \beta$ line- by the letter "h". Non-magnetic standard stars in the table are marked with the symbol "*”, standard stars with a well-known variability law of the longitudinal field component are denoted by "**". The stars for which the magnetic field was discovered for the first time are printed in italics. 
The results of measurements of magnetic fields, radial velocities, and rotation velocities of stars according to observations 2010

\begin{tabular}{|c|c|c|c|c|c|}
\hline Star & JD $(2450000+)$ & $\begin{array}{c}B_{e} \pm \sigma \\
\mathrm{G}\end{array}$ & $\begin{array}{l}V_{r} \pm \sigma \\
\mathrm{km} \mathrm{s}^{-1}\end{array}$ & $\begin{array}{c}v_{e} \sin i \pm \sigma \\
\mathrm{km} \mathrm{s}^{-1}\end{array}$ & $S / N$ \\
\hline (1) & $(2)$ & (3) & (4) & $(5)$ & (6) \\
\hline HD 653 & $\begin{array}{l}5488.445 \\
5554.220\end{array}$ & $\begin{array}{l}-30 \pm 110(\mathrm{z}) \\
-100 \pm 140(\mathrm{r}) \\
+130 \pm 110(\mathrm{z}) \\
+320 \pm 80(\mathrm{r})\end{array}$ & $\begin{array}{l}+30.2 \pm 2.6 \\
-17.0 \pm 3.4\end{array}$ & $\begin{array}{l}80 \pm 13 \\
70 \pm 10\end{array}$ & 220 \\
\hline HD 965 & $\begin{array}{l}5431.458 \\
5459.492 \\
5461.462 \\
5553.174\end{array}$ & $\begin{array}{l}-70 \pm 20(\mathrm{z}) \\
-240 \pm 50(\mathrm{z}) \\
-140 \pm 50(\mathrm{z}) \\
-340 \pm 20(\mathrm{z}) \\
-330 \pm 40(\mathrm{r}) \\
-360 \pm 30(\mathrm{z}) \\
-330 \pm 20(\mathrm{r})\end{array}$ & $\begin{array}{l}-1.0 \pm 1.8 \\
-4.5 \pm 2.6 \\
-3.4 \pm 2.9 \\
-1.3 \pm 1.0 \\
-3.2 \pm 2.4\end{array}$ & $<20$ & $\begin{array}{l}170 \\
200 \\
230 \\
200\end{array}$ \\
\hline HD 5441 & 5488.479 & $\begin{array}{l}-440 \pm 20(\mathrm{z}) \\
-450 \pm 20(\mathrm{r})\end{array}$ & $+43.6 \pm 2.4$ & $21 \pm 3$ & 190 \\
\hline HD 5797 & $\begin{array}{l}5253.628 \\
5255.218\end{array}$ & & $-3.3 \pm 1.1$ & $<20$ & 220 \\
\hline HD 6757 & 5431.550 & $+2800 \pm 90(\mathrm{z})$ & $-9.4 \pm 2.4$ & $28 \pm 5$ & 330 \\
\hline HD 16705 & 5553.192 & $\begin{array}{c}-3720 \pm 1660(\mathrm{z}) \\
+720 \pm 200(\mathrm{r})\end{array}$ & $-12.2 \pm 2.9$ & $100 \pm 8$ & 350 \\
\hline HD 17330 & 5553.225 & $\begin{array}{l}+150 \pm 320(\mathrm{z}) \\
-420 \pm 30(\mathrm{r})\end{array}$ & $-13.6 \pm 2.8$ & $<20$ & 400 \\
\hline HD 23924 & 5554.435 & $\begin{array}{l}-60 \pm 50(\mathrm{z}) \\
-50 \pm 60(\mathrm{r})\end{array}$ & $-2.1 \pm 2.0$ & $40 \pm 6$ & 300 \\
\hline HD 23964 & 5555.475 & $-10 \pm 30(\mathrm{z})$ & $+10.2 \pm 1.9$ & $20 \pm 3$ & 250 \\
\hline HD 29762 & 5555.456 & $\begin{array}{l}+300 \pm 50(\mathrm{z}) \\
+190 \pm 60(\mathrm{r})\end{array}$ & $-9.2 \pm 1.7$ & $32 \pm 4$ & 250 \\
\hline HD 32549 & $\begin{array}{l}5488.619 \\
5554.495\end{array}$ & $\begin{array}{l}+140 \pm 80(\mathrm{z}) \\
+60 \pm 70(\mathrm{r}) \\
-250 \pm 120(\mathrm{z}) \\
+280 \pm 150(\mathrm{r})\end{array}$ & $\begin{array}{l}+24.3 \pm 2.8 \\
+10.0 \pm 2.9\end{array}$ & $61 \pm 6$ & 600 \\
\hline HD $33256^{*}$ & 5202.267 & $\begin{array}{c}+20 \pm 20(\mathrm{z}) \\
+110 \pm 160(\mathrm{~h})\end{array}$ & $-21.2 \pm 2.4$ & $<20$ & 500 \\
\hline
\end{tabular}


Contd.

\begin{tabular}{|c|c|c|c|c|c|}
\hline Star & JD $(2450000+)$ & $\begin{array}{c}B_{e} \pm \sigma \\
\mathrm{G}\end{array}$ & $\begin{array}{l}V_{r} \pm \sigma \\
\mathrm{km} \mathrm{s}^{-1}\end{array}$ & $\begin{array}{c}v_{e} \sin i \pm \sigma \\
\mathrm{km} \mathrm{s}^{-1}\end{array}$ & $S / N$ \\
\hline (1) & $(2)$ & (3) & (4) & (5) & (6) \\
\hline & 5488.571 & $\begin{array}{l}-10 \pm 10(\mathrm{z}) \\
-10 \pm 10(\mathrm{r})\end{array}$ & $+14.6 \pm 2.5$ & & 400 \\
\hline & 5554.253 & $\begin{array}{l}-50 \pm 10(\mathrm{z}) \\
-50 \pm 10(\mathrm{r})\end{array}$ & $+3.6 \pm 1.5$ & & 500 \\
\hline & 5555.245 & $\begin{array}{r}0 \pm 10(\mathrm{z}) \\
+10 \pm 10(\mathrm{r})\end{array}$ & $+6.0 \pm 3.1$ & & 800 \\
\hline HD 34307 & 5553.313 & $\begin{array}{c}-50 \pm 90(\mathrm{z}) \\
+160 \pm 180(\mathrm{r})\end{array}$ & $+29.8 \pm 2.8$ & $21 \pm 4$ & 300 \\
\hline HD 34968 & 5553.395 & $\begin{array}{r}+30 \pm 250(\mathrm{z}) \\
-170 \pm 240(\mathrm{r})\end{array}$ & $+27.9 \pm 3.8$ & $105 \pm 20$ & 300 \\
\hline HD 35101 & 5553.577 & $\begin{array}{r}+600 \pm 950(\mathrm{z}) \\
-30 \pm 190(\mathrm{r})\end{array}$ & $-18.9 \pm 2.8$ & $110 \pm 30$ & 400 \\
\hline & 5554.592 & $\begin{array}{l}+740 \pm 530(\mathrm{z}) \\
-430 \pm 190(\mathrm{r})\end{array}$ & $-25.7 \pm 2.5$ & & 400 \\
\hline HD 35298 & 5554.300 & $\begin{array}{l}-6090 \pm 300(\mathrm{z}) \\
-3440 \pm 150(\mathrm{r})\end{array}$ & $+20.9 \pm 3.4$ & $50 \pm 7$ & 400 \\
\hline HD 35379 & 5552.565 & $\begin{array}{l}-200 \pm 110(\mathrm{z}) \\
-250 \pm 120(\mathrm{r})\end{array}$ & $+3.7 \pm 2.8$ & $45 \pm 5$ & 300 \\
\hline HD 35456 & 5554.338 & $\begin{array}{l}+650 \pm 70(\mathrm{z}) \\
+640 \pm 80(\mathrm{r})\end{array}$ & $+11.0 \pm 2.8$ & $22 \pm 2$ & 400 \\
\hline HD 35548 & 5553.294 & $\begin{array}{l}-10 \pm 20(\mathrm{z}) \\
+20 \pm 60(\mathrm{r})\end{array}$ & $-9.3 \pm 2.4$ & $<20$ & 500 \\
\hline HD 35575 & 5553.304 & $\begin{array}{l}-200 \pm 490(\mathrm{z}) \\
+310 \pm 270(\mathrm{r})\end{array}$ & $+24.2 \pm 2.9$ & $150 \pm 10$ & 500 \\
\hline HD 35730 & 5553.238 & $\begin{array}{r}+150 \pm 320(\mathrm{z}) \\
-30 \pm 220(\mathrm{r})\end{array}$ & $+22.7 \pm 2.7$ & $54 \pm 6$ & 400 \\
\hline HD 35881 & 5553.258 & $\begin{array}{l}-1070 \pm 590(\mathrm{z}) \\
-1130 \pm 370(\mathrm{r})\end{array}$ & $+19.5 \pm 3.7$ & $205 \pm 20$ & 500 \\
\hline HD 36032 & 5553.454 & $\begin{array}{l}-900 \pm 200(\mathrm{z}) \\
-110 \pm 230(\mathrm{r})\end{array}$ & $+29.6 \pm 3.4$ & $205 \pm 16$ & 300 \\
\hline HD 36313 & 5554.320 & $\begin{array}{l}+120 \pm 120(\mathrm{z}) \\
+560 \pm 180(\mathrm{r})\end{array}$ & $+43.0 \pm 2.9$ & $27 \pm 2$ & 400 \\
\hline
\end{tabular}


Contd.

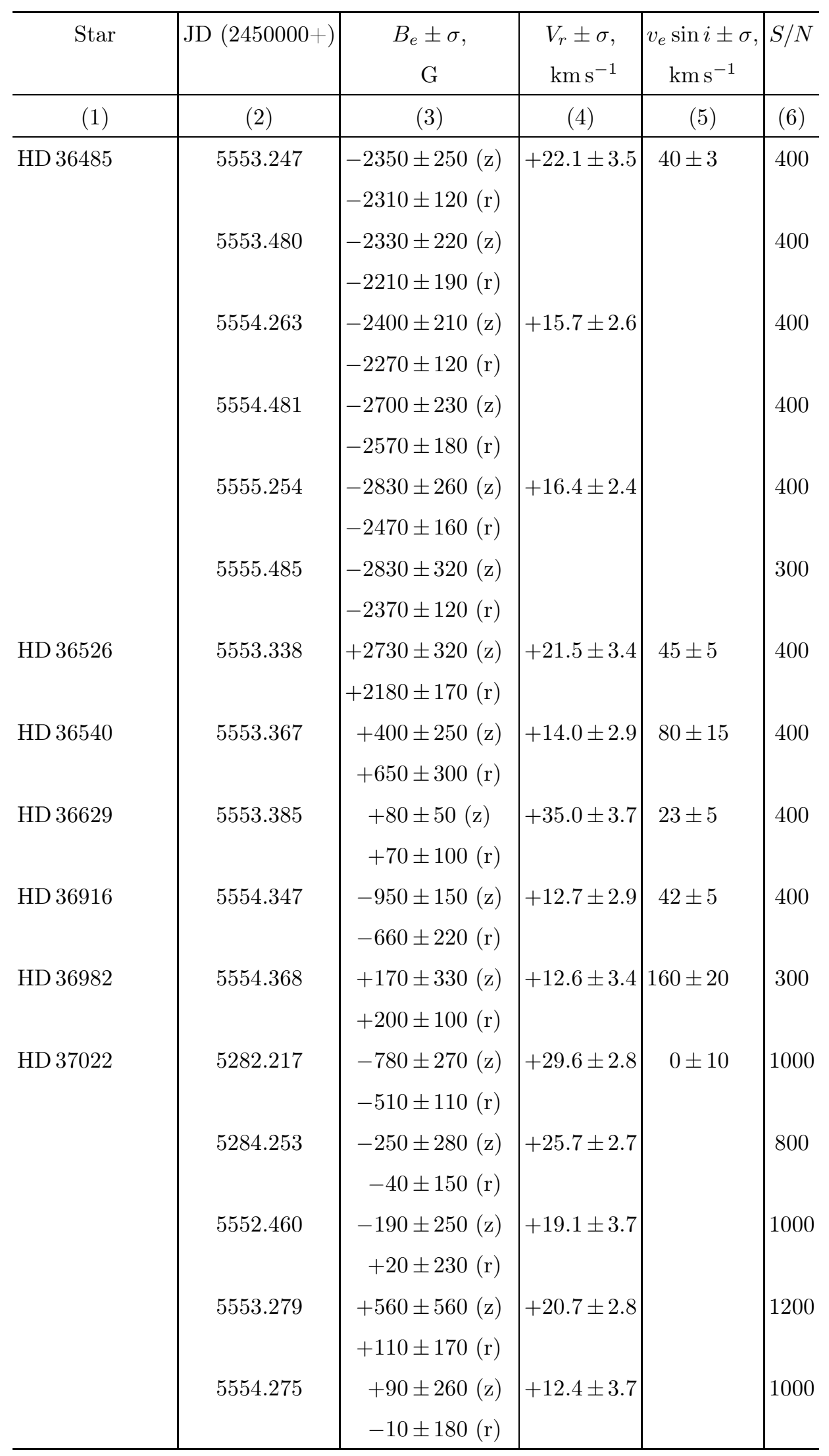


Contd.

\begin{tabular}{|c|c|c|c|c|c|}
\hline Star & JD $(2450000+)$ & $\begin{array}{c}B_{e} \pm \sigma \\
\mathrm{G}\end{array}$ & $\begin{array}{l}V_{r} \pm \sigma \\
\mathrm{km} \mathrm{s}^{-1}\end{array}$ & $\begin{array}{c}v_{e} \sin i \pm \sigma \\
\mathrm{km} \mathrm{s}^{-1}\end{array}$ & $S / N$ \\
\hline (1) & $(2)$ & (3) & $(4)$ & (5) & (6) \\
\hline & 5555.270 & $\begin{array}{r}+630 \pm 170(\mathrm{z}) \\
-80 \pm 180(\mathrm{r})\end{array}$ & $+16.2 \pm 3.7$ & & 1000 \\
\hline HD 37140 & 5555.298 & $\begin{array}{l}-580 \pm 90(\mathrm{z}) \\
-350 \pm 90(\mathrm{r})\end{array}$ & $+23.4 \pm 2.4$ & $25 \pm 2$ & 400 \\
\hline HD 37151 & 5555.503 & $\begin{array}{r}0 \pm 80(\mathrm{z}) \\
+30 \pm 50(\mathrm{r})\end{array}$ & $+18.2 \pm 2.7$ & $30 \pm 3$ & 400 \\
\hline HD 37479 & 5555.325 & $\begin{array}{c}-1050 \pm 1080(\mathrm{z}) \\
+140 \pm 330(\mathrm{r})\end{array}$ & $+15.0 \pm 2.9$ & $100 \pm 25$ & 400 \\
\hline HD 37525 & 5555.342 & $\begin{array}{r}+670 \pm 1670(\mathrm{z}) \\
+20 \pm 290(\mathrm{r})\end{array}$ & $+39.0 \pm 3.4$ & $150 \pm 30$ & 400 \\
\hline HD 37687 & 5555.507 & $\begin{array}{l}+580 \pm 40(\mathrm{z}) \\
+490 \pm 40(\mathrm{r})\end{array}$ & $+18.1 \pm 2.3$ & $22 \pm 3$ & 400 \\
\hline HD 37776 & 5282.183 & $\begin{array}{l}+8600 \pm 1700(\mathrm{z}) \\
+2090 \pm 170(\mathrm{r})\end{array}$ & $+26.5 \pm 3.1$ & & 600 \\
\hline & 5284.233 & $\begin{array}{c}-6200 \pm 7100(\mathrm{z}) \\
-130 \pm 290(\mathrm{r})\end{array}$ & $+23.6 \pm 3.7$ & & 500 \\
\hline HD 38104 & 5202.600 & $\begin{array}{l}+160 \pm 110(\mathrm{z}) \\
-110 \pm 210(\mathrm{~h})\end{array}$ & $+3.8 \pm 1.1$ & $35 \pm 5$ & 700 \\
\hline & 5288.306 & $\begin{aligned} 0 & \pm 50(\mathrm{z}) \\
+100 & \pm 280(\mathrm{~h})\end{aligned}$ & $-2.4 \pm 1.4$ & & 500 \\
\hline HD 38823 & 5202.600 & $\begin{array}{l}-220 \pm 80(\mathrm{z}) \\
-280 \pm 150(\mathrm{~h})\end{array}$ & $+3.6 \pm 1.2$ & $36 \pm 3$ & 300 \\
\hline HD 39317 & 5554.500 & $\begin{array}{l}+10 \pm 110(\mathrm{z}) \\
+50 \pm 160(\mathrm{r})\end{array}$ & $-12.1 \pm 2.6$ & $70 \pm 15$ & 500 \\
\hline \multirow[t]{4}{*}{ HD 45583} & 5202.348 & $\begin{array}{l}-2400 \pm 200(\mathrm{z}) \\
-1650 \pm 260(\mathrm{~h})\end{array}$ & $+21.4 \pm 2.8$ & $80 \pm 10$ & 400 \\
\hline & 5284.272 & $\begin{array}{l}+5320 \pm 470(\mathrm{z}) \\
+2700 \pm 100(\mathrm{r})\end{array}$ & $+24.8 \pm 3.8$ & & 300 \\
\hline & 5488.592 & $\begin{array}{l}+5710 \pm 620(\mathrm{z}) \\
+2160 \pm 170(\mathrm{r})\end{array}$ & $+32.3 \pm 2.7$ & & 500 \\
\hline & 5552.485 & $\begin{array}{l}+2770 \pm 290(\mathrm{z}) \\
+2650 \pm 190(\mathrm{r})\end{array}$ & $+23.4 \pm 2.8$ & & 400 \\
\hline
\end{tabular}


Contd.

\begin{tabular}{|c|c|c|c|c|c|}
\hline Star & JD $(2450000+)$ & $\begin{array}{c}B_{e} \pm \sigma \\
\mathrm{G}\end{array}$ & $\begin{array}{l}V_{r} \pm \sigma \\
\mathrm{km} \mathrm{s}^{-1}\end{array}$ & $\begin{array}{c}v_{e} \sin i \pm \sigma \\
\mathrm{km} \mathrm{s}^{-1}\end{array}$ & $S / N$ \\
\hline (1) & $(2)$ & $(3)$ & $(4)$ & (5) & (6) \\
\hline \multirow{4}{*}{$H D 49884$} & 5553.425 & $\begin{array}{l}+4807 \pm 403(\mathrm{z}) \\
+2670 \pm 190(\mathrm{r})\end{array}$ & $+30.0 \pm 2.9$ & \multirow{4}{*}{$<20$} & \multirow[b]{2}{*}{300} \\
\hline & 5283.302 & $\begin{array}{l}-100 \pm 50(\mathrm{z}) \\
-180 \pm 30(\mathrm{r})\end{array}$ & $-6.9 \pm 2.7$ & & \\
\hline & 5284.297 & $\begin{array}{l}-190 \pm 40(\mathrm{z}) \\
-125 \pm 10(\mathrm{r})\end{array}$ & $+0.3 \pm 0.1$ & & 400 \\
\hline & 5552.625 & $\begin{array}{l}-310 \pm 40(\mathrm{z}) \\
-290 \pm 30(\mathrm{r})\end{array}$ & $-5.5 \pm 2.7$ & & 200 \\
\hline HD 50169 & 5282.238 & $\begin{array}{r}+130 \pm 50(\mathrm{z}) \\
+80 \pm 15(\mathrm{r})\end{array}$ & $+14.6 \pm 2.4$ & $<20$ & 300 \\
\hline HD 50461 & 5554.418 & $\begin{array}{r}+1550 \pm 770(\mathrm{z}) \\
+290 \pm 220(\mathrm{r})\end{array}$ & $+29.5 \pm 3.4$ & $80 \pm 15$ & 400 \\
\hline HD 51418 & $\begin{array}{l}5281.438 \\
5282.306\end{array}$ & $\begin{array}{l}+520 \pm 50(\mathrm{z}) \\
-460 \pm 70(\mathrm{z}) \\
-520 \pm 40(\mathrm{r})\end{array}$ & $\begin{array}{l}-28.3 \pm 2.6 \\
-33.2 \pm 2.7\end{array}$ & $28 \pm 5$ & $\begin{array}{l}300 \\
700\end{array}$ \\
\hline & 5283.318 & $\begin{array}{l}+360 \pm 60(\mathrm{z}) \\
+320 \pm 30(\mathrm{r})\end{array}$ & $-30.7 \pm 3.1$ & & 400 \\
\hline & 5284.354 & $\begin{array}{l}-250 \pm 40(\mathrm{z}) \\
-200 \pm 20(\mathrm{r})\end{array}$ & $-25.1 \pm 2.4$ & & 500 \\
\hline HD 52711 & 5288.294 & $-40 \pm 20(\mathrm{z})$ & $+30.9 \pm 2.7$ & $33 \pm 5$ & 400 \\
\hline$H D 54824$ & 5283.279 & $\begin{array}{l}-690 \pm 180(\mathrm{z}) \\
-482 \pm 50(\mathrm{r})\end{array}$ & $+27.0 \pm 2.8$ & $50 \pm 10$ & 300 \\
\hline & 5552.604 & $\begin{array}{l}+470 \pm 120(\mathrm{z}) \\
+200 \pm 160(\mathrm{r})\end{array}$ & $+29.7 \pm 2.9$ & & 350 \\
\hline & 5553.598 & $\begin{array}{l}-630 \pm 70(\mathrm{z}) \\
-470 \pm 110(\mathrm{r})\end{array}$ & $+35.5 \pm 2.9$ & & 300 \\
\hline HD 62512 & 5553.545 & $\begin{array}{r}-80 \pm 50(\mathrm{z}) \\
-100 \pm 50(\mathrm{r})\end{array}$ & $+12.0 \pm 2.8$ & $22 \pm 5$ & 300 \\
\hline & 5554.518 & $\begin{array}{l}-180 \pm 40(\mathrm{z}) \\
-200 \pm 70(\mathrm{r})\end{array}$ & $+4.2 \pm 3.6$ & & 300 \\
\hline HD $65339^{* *}$ & 5202.608 & $\begin{array}{l}+3740 \pm 100(\mathrm{z}) \\
+2740 \pm 300(\mathrm{~h})\end{array}$ & $-1.2 \pm 1.0$ & $25 \pm 5$ & 600 \\
\hline
\end{tabular}


Contd.

\begin{tabular}{|c|c|c|c|c|c|}
\hline Star & JD $(2450000+)$ & $\begin{array}{c}B_{e} \pm \sigma \\
\mathrm{G}\end{array}$ & $\begin{array}{l}V_{r} \pm \sigma, \\
\mathrm{km} \mathrm{s}^{-1}\end{array}$ & $\begin{array}{c}v_{e} \sin i \pm \sigma \\
\mathrm{km} \mathrm{s}^{-1}\end{array}$ & $S / N$ \\
\hline \multirow[t]{15}{*}{ (1) } & (2) & (3) & (4) & (5) & (6) \\
\hline & 5281.451 & $+2340 \pm 190(\mathrm{z})$ & $-2.4 \pm 1.7$ & & 600 \\
\hline & 5282.285 & $-3190 \pm 120(\mathrm{z})$ & $-5.7 \pm 2.4$ & & 600 \\
\hline & & $-3080 \pm 50(\mathrm{r})$ & & & \\
\hline & 5283.330 & $+3520 \pm 170(\mathrm{z})$ & $-8.6 \pm 3.7$ & & 500 \\
\hline & & $+3350 \pm 40(\mathrm{r})$ & & & \\
\hline & 5284.329 & $+3460 \pm 170(\mathrm{z})$ & $-2.4 \pm 2.1$ & & 500 \\
\hline & & $+3030 \pm 50(\mathrm{r})$ & & & \\
\hline & 5311.318 & $-6450 \pm 150(\mathrm{z})$ & $-7.7 \pm 2.9$ & & 500 \\
\hline & & $-3630 \pm 250(\mathrm{~h})$ & & & \\
\hline & 5315.335 & $+3990 \pm 90(\mathrm{z})$ & $-2.1 \pm 2.0$ & & 400 \\
\hline & & $+2450 \pm 250(\mathrm{~h})$ & & & \\
\hline & 5348.260 & $+4050 \pm 120(\mathrm{z})$ & $-3.2 \pm 1.3$ & & 400 \\
\hline & & $+3700 \pm 40(\mathrm{r})$ & & & \\
\hline & 5552.638 & $-5580 \pm 190(\mathrm{z})$ & $-11.7 \pm 2.9$ & & 400 \\
\hline \multirow[t]{5}{*}{ HD 89069} & 5202.658 & $-400 \pm 50(\mathrm{z})$ & $-2.5 \pm 1.1$ & $<20$ & 400 \\
\hline & 5311.381 & $-720 \pm 30(\mathrm{z})$ & $-12.7 \pm 2.8$ & & 300 \\
\hline & & $-260 \pm 240(\mathrm{~h})$ & & & \\
\hline & 5315.395 & $-250 \pm 20(\mathrm{z})$ & $-7.8 \pm 2.9$ & & 200 \\
\hline & & $-260 \pm 30(\mathrm{r})$ & & & \\
\hline \multirow[t]{6}{*}{ HD 90763} & 5552.668 & $+180 \pm 80(\mathrm{z})$ & $-26.0 \pm 3.8$ & $50 \pm 5$ & 400 \\
\hline & & $-40 \pm 60(\mathrm{r})$ & & & \\
\hline & 5553.592 & $-120 \pm 110(\mathrm{z})$ & $-25.5 \pm 3.4$ & & 500 \\
\hline & & $+100 \pm 100(\mathrm{r})$ & & & \\
\hline & 5554.625 & $+170 \pm 70(\mathrm{z})$ & $-32.7 \pm 3.6$ & & 500 \\
\hline & & $-220 \pm 130(\mathrm{r})$ & & & \\
\hline \multirow[t]{4}{*}{ HD 93294} & 5202.560 & $-40 \pm 50(\mathrm{z})$ & $+25.7 \pm 2.6$ & $<20$ & 400 \\
\hline & & $-30 \pm 310(\mathrm{~h})$ & & & \\
\hline & 5348.248 & $-90 \pm 20(\mathrm{z})$ & $+21.3 \pm 2.1$ & & 300 \\
\hline & & $-80 \pm 20(\mathrm{r})$ & & & \\
\hline HD 96003 & 5345.256 & $-210 \pm 20(\mathrm{z})$ & $-13.7 \pm 2.4$ & $<20$ & 600 \\
\hline \multirow[t]{2}{*}{ HD 97633} & 5552.675 & $+40 \pm 20(\mathrm{z})$ & $-4.9 \pm 2.5$ & $25 \pm 5$ & 1500 \\
\hline & & $-30 \pm 70(\mathrm{r})$ & & & \\
\hline
\end{tabular}


Contd.

\begin{tabular}{|c|c|c|c|c|c|}
\hline Star & JD $(2450000+)$ & $\begin{array}{c}B_{e} \pm \sigma \\
\mathrm{G}\end{array}$ & $\begin{array}{l}V_{r} \pm \sigma \\
\mathrm{kms} \mathrm{s}^{-1}\end{array}$ & $\begin{array}{c}v_{e} \sin i \pm \sigma \\
\mathrm{km} \mathrm{s}^{-1}\end{array}$ & $S / N$ \\
\hline (1) & $(2)$ & $(3)$ & $(4)$ & (5) & (6) \\
\hline \multirow{5}{*}{ HD 108506} & 5553.673 & $\begin{array}{l}+10 \pm 20(\mathrm{z}) \\
-20 \pm 40(\mathrm{r})\end{array}$ & $-1.0 \pm 2.9$ & & 1000 \\
\hline & 5554.640 & $\begin{array}{r}+120 \pm 30(\mathrm{z}) \\
+40 \pm 50(\mathrm{r})\end{array}$ & $-7.5 \pm 2.6$ & & 1500 \\
\hline & 5553.708 & $\begin{array}{l}+370 \pm 510(\mathrm{z}) \\
-560 \pm 330(\mathrm{r})\end{array}$ & $0.0 \pm 1.6$ & $150 \pm 20$ & 400 \\
\hline & 5554.633 & $\begin{array}{r}+160 \pm 250(\mathrm{z}) \\
+50 \pm 230(\mathrm{r})\end{array}$ & $-16.2 \pm 3.4$ & & 500 \\
\hline & 5555.566 & $\begin{array}{r}-60 \pm 510(\mathrm{z}) \\
-580 \pm 250(\mathrm{r})\end{array}$ & $-5.0 \pm 3.9$ & & 400 \\
\hline HD 110066 & 5345.244 & $-220 \pm 10(\mathrm{z})$ & $-13.9 \pm 2.7$ & $<20$ & 500 \\
\hline HD $112413^{* *}$ & 5202.581 & $\begin{array}{l}-880 \pm 50(\mathrm{z}) \\
-680 \pm 70(\mathrm{~h})\end{array}$ & $+7.1 \pm 2.4$ & & 3000 \\
\hline \multirow{5}{*}{ HD 113894} & 5281.468 & $+860 \pm 80(\mathrm{z})$ & $+1.9 \pm 0.8$ & & 3000 \\
\hline & 5345.230 & $-820 \pm 59(\mathrm{z})$ & $-1.7 \pm 1.3$ & & 2000 \\
\hline & 5553.645 & $\begin{array}{l}+990 \pm 40(\mathrm{z}) \\
+900 \pm 30(\mathrm{r})\end{array}$ & $+8.2 \pm 2.4$ & $23 \pm 3$ & 300 \\
\hline & 5554.650 & $\begin{array}{l}+840 \pm 40(\mathrm{z}) \\
+870 \pm 30(\mathrm{r})\end{array}$ & $+1.6 \pm 2.6$ & & 300 \\
\hline & 5555.579 & $\begin{array}{l}+760 \pm 30(\mathrm{z}) \\
+690 \pm 60(\mathrm{r})\end{array}$ & $+4.6 \pm 2.1$ & & 300 \\
\hline HD 114125 & 5282.42 & $\begin{array}{l}+20 \pm 80(\mathrm{z}) \\
-50 \pm 40(\mathrm{r})\end{array}$ & $-56.1 \pm 3.1$ & $25 \pm 5$ & 400 \\
\hline & 5345.279 & $+20 \pm 50(\mathrm{z})$ & $-59.7 \pm 2.4$ & & 300 \\
\hline$H D 118054$ & 5553.658 & $\begin{array}{r}-420 \pm 190(\mathrm{z}) \\
0 \pm 140(\mathrm{r})\end{array}$ & $-10.4 \pm 2.5$ & $65 \pm 5$ & 300 \\
\hline \multirow{3}{*}{ HD 135297} & 5554.662 & $\begin{array}{l}-580 \pm 130(\mathrm{z}) \\
-890 \pm 210(\mathrm{r})\end{array}$ & $-17.8 \pm 2.6$ & & \\
\hline & 5282.405 & $\begin{array}{l}+50 \pm 50(\mathrm{z}) \\
+70 \pm 30(\mathrm{r})\end{array}$ & $-44.5 \pm 2.9$ & $27 \pm 5$ & 500 \\
\hline & 5345.324 & $-170 \pm 30(\mathrm{z})$ & $-41.3 \pm 2.6$ & & 400 \\
\hline HD 135679 & 5555.591 & $+1120 \pm 40(\mathrm{z})$ & $+0.8 \pm 2.1$ & $<20$ & 410 \\
\hline
\end{tabular}


Contd.

\begin{tabular}{|c|c|c|c|c|c|}
\hline Star & $\mathrm{JD}(2450000+)$ & $\begin{array}{c}B_{e} \pm \sigma \\
\mathrm{G}\end{array}$ & $\begin{array}{l}V_{r} \pm \sigma \\
\mathrm{km} \mathrm{s}^{-1}\end{array}$ & $\begin{array}{c}v_{e} \sin i \pm \sigma \\
\mathrm{km} \mathrm{s}^{-1}\end{array}$ & $S / N$ \\
\hline (1) & (2) & (3) & (4) & (5) & (6) \\
\hline HD $137909^{* *}$ & 5202.590 & $\begin{array}{l}+960 \pm 24(\mathrm{r}) \\
+430 \pm 50(\mathrm{z}) \\
+270 \pm 70(\mathrm{~h})\end{array}$ & $+9.2 \pm 1.1$ & $<20$ & 1800 \\
\hline HD 138633 & 5282.470 & $\begin{array}{l}+310 \pm 30(\mathrm{z}) \\
+350 \pm 20(\mathrm{r})\end{array}$ & $-14.6 \pm 2.8$ & $<20$ & 300 \\
\hline & 5287.489 & $\begin{array}{l}-290 \pm 20(\mathrm{z}) \\
-210 \pm 370(\mathrm{~h})\end{array}$ & $-4.7 \pm 2.1$ & & 300 \\
\hline HD 138777 & 5348.300 & $\begin{array}{l}+2150 \pm 60(\mathrm{z}) \\
+2000 \pm 40(\mathrm{r})\end{array}$ & $-46.3 \pm 2.7$ & $25 \pm 5$ & 250 \\
\hline HD 149046 & 5345.335 & $-105 \pm 150(\mathrm{z})$ & $-28.3 \pm 3.8$ & $25 \pm 5$ & 250 \\
\hline HD $152107^{* *}$ & 5287.450 & $\begin{array}{l}+1180 \pm 40(\mathrm{z}) \\
+290 \pm 300(\mathrm{~h})\end{array}$ & $+7.2 \pm 2.1$ & $30 \pm 5$ & 1000 \\
\hline HD 157740 & 5555.669 & $\begin{array}{l}+130 \pm 70(\mathrm{z}) \\
+130 \pm 60(\mathrm{r})\end{array}$ & $+6.5 \pm 3.1$ & $25 \pm 5$ & 300 \\
\hline HD 158450 & 5287.518 & $\begin{array}{l}-4280 \pm 110(\mathrm{z}) \\
-2320 \pm 420(\mathrm{~h})\end{array}$ & $-12.7 \pm 2.4$ & $27 \pm 5$ & 300 \\
\hline & 5315.420 & $\begin{array}{l}-4430 \pm 150(\mathrm{z}) \\
-3640 \pm 40(\mathrm{r})\end{array}$ & $-16.4 \pm 2.8$ & & 250 \\
\hline & 5345.367 & $-100 \pm 65(\mathrm{z})$ & $-19.6 \pm 2.7$ & & 300 \\
\hline & 5348.435 & $\begin{array}{l}-4480 \pm 130(\mathrm{z}) \\
-3900 \pm 50(\mathrm{r})\end{array}$ & $-18.2 \pm 2.4$ & & 250 \\
\hline HD 158974* & 5287.458 & $\begin{array}{l}+20 \pm 10(\mathrm{z}) \\
+60 \pm 460(\mathrm{~h})\end{array}$ & $-23.6 \pm 2.7$ & $25 \pm 5$ & 400 \\
\hline & 5345.385 & $-20 \pm 10(\mathrm{z})$ & $-34.3 \pm 3.4$ & & 600 \\
\hline & 5406.468 & $-10 \pm 10(\mathrm{z})$ & $-30.3 \pm 2.9$ & & 400 \\
\hline HD 168856 & 5287.581 & $\begin{array}{r}+360 \pm 190(\mathrm{z}) \\
-70 \pm 280(\mathrm{~h})\end{array}$ & $-21.8 \pm 2.6$ & & 300 \\
\hline & 5315.562 & $\begin{array}{r}-1110 \pm 320(\mathrm{z}) \\
-250 \pm 180(\mathrm{r})\end{array}$ & & & 400 \\
\hline HD 169191* & $\begin{array}{l}5460.252 \\
5461.284 \\
5553.102\end{array}$ & $\begin{array}{r}-20 \pm 10(\mathrm{z}) \\
+142 \pm 10(\mathrm{z}) \\
0 \pm 10(\mathrm{z})\end{array}$ & $\begin{array}{l}-17.0 \pm 2.8 \\
-22.0 \pm 2.9 \\
-14.0 \pm 2.8\end{array}$ & $<20$ & $\begin{array}{l}500 \\
500 \\
500\end{array}$ \\
\hline
\end{tabular}


Contd.

\begin{tabular}{|c|c|c|c|c|c|}
\hline Star & JD $(2450000+)$ & $\begin{array}{c}B_{e} \pm \sigma \\
\mathrm{G}\end{array}$ & $\begin{array}{l}V_{r} \pm \sigma \\
\mathrm{km} \mathrm{s}^{-1}\end{array}$ & $\begin{array}{c}v_{e} \sin i \pm \sigma \\
\mathrm{km} \mathrm{s}^{-1}\end{array}$ & $S / N$ \\
\hline$(1)$ & $(2)$ & $(3)$ & (4) & $(5)$ & (6) \\
\hline \multirow{5}{*}{ HD 178308} & \multirow{3}{*}{5348.348} & $+10 \pm 10(\mathrm{r})$ & & \multirow{5}{*}{$75 \pm 10$} & \multirow{3}{*}{400} \\
\hline & & $+20 \pm 120(\mathrm{z})$ & $-24.8 \pm 2.9$ & & \\
\hline & & $-140 \pm 160(\mathrm{r})$ & & & \\
\hline & 5554.125 & $+240 \pm 260(\mathrm{z})$ & $-25.7 \pm 2.6$ & & 400 \\
\hline & & $+130 \pm 130(\mathrm{r})$ & & & \\
\hline \multirow[t]{2}{*}{ HD 178892} & 5460.235 & $+4580 \pm 200(\mathrm{z})$ & $-10.9 \pm 2.7$ & \multirow[t]{2}{*}{$30 \pm 5$} & 400 \\
\hline & 5461.268 & $+2790 \pm 170(\mathrm{z})$ & $-17.2 \pm 3.8$ & & 300 \\
\hline \multirow[t]{2}{*}{ HD 182180} & \multirow[t]{2}{*}{5348.500} & $-4000 \pm 1200(\mathrm{z})$ & $+5.4 \pm 2.8$ & \multirow[t]{2}{*}{$200 \pm 30$} & \multirow[t]{2}{*}{500} \\
\hline & & $-750 \pm 310(\mathrm{r})$ & & & \\
\hline \multirow[t]{2}{*}{ HD 198920} & \multirow[t]{2}{*}{5406.527} & $+10 \pm 20(\mathrm{z})$ & $+18.7 \pm 3.4$ & \multirow[t]{2}{*}{$30 \pm 5$} & \multirow[t]{2}{*}{300} \\
\hline & & $+10 \pm 10(\mathrm{r})$ & & & \\
\hline \multirow[t]{2}{*}{ HD 199180} & \multirow[t]{2}{*}{5287.592} & $-340 \pm 60(\mathrm{z})$ & $-17.3 \pm 2.8$ & \multirow[t]{2}{*}{$27 \pm 5$} & \multirow[t]{2}{*}{300} \\
\hline & & $-350 \pm 300(\mathrm{~h})$ & & & \\
\hline \multirow[t]{7}{*}{ HD 201174} & 5345.407 & $+1810 \pm 90(\mathrm{z})$ & $-13.8 \pm 3.4$ & \multirow{7}{*}{$25 \pm 5$} & 400 \\
\hline & 5459.442 & $+710 \pm 90(\mathrm{z})$ & $-10.6 \pm 2.7$ & & 300 \\
\hline & 5460.193 & $+2110 \pm 50(\mathrm{z})$ & $-4.6 \pm 2.8$ & & 300 \\
\hline & \multirow[t]{2}{*}{5554.190} & $+680 \pm 100(\mathrm{z})$ & $-16.5 \pm 3.4$ & & \multirow[t]{2}{*}{300} \\
\hline & & $+480 \pm 60(\mathrm{r})$ & & & \\
\hline & 5555.159 & $+2070 \pm 80(\mathrm{z})$ & $-9.9 \pm 3.4$ & & 350 \\
\hline & & $+1930 \pm 40(\mathrm{r})$ & & & \\
\hline \multirow[t]{11}{*}{ HD $201601^{* *}$} & 5287.600 & $-1190 \pm 30(\mathrm{z})$ & $-18.3 \pm 2.0$ & \multirow[t]{11}{*}{$<20$} & \multirow[t]{2}{*}{1000} \\
\hline & & $-630 \pm 460(\mathrm{~h})$ & & & \\
\hline & 5431.480 & $-1230 \pm 30(\mathrm{z})$ & $-14.9 \pm 2.7$ & & 700 \\
\hline & 5460.218 & $-1050 \pm 30(\mathrm{z})$ & $-20.3 \pm 2.9$ & & 1000 \\
\hline & 5461.250 & $-1070 \pm 50(\mathrm{z})$ & $-19.1 \pm 2.8$ & & 1000 \\
\hline & 5488.357 & $-1020 \pm 50(\mathrm{z})$ & $-14.4 \pm 3.8$ & & 600 \\
\hline & 5553.160 & $-1130 \pm 30(\mathrm{z})$ & $-16.6 \pm 2.7$ & & 900 \\
\hline & & $-1000 \pm 30(\mathrm{r})$ & & & \\
\hline & 5554.100 & $-1050 \pm 40(\mathrm{z})$ & $-16.3 \pm 2.6$ & & 1000 \\
\hline & 5555.100 & $-1120 \pm 40(\mathrm{z})$ & $-16.7 \pm 2.4$ & & 1000 \\
\hline & & $-960 \pm 30(\mathrm{r})$ & & & \\
\hline HD 217401 & 5488.381 & $-30 \pm 70(\mathrm{z})$ & $+1.4 \pm 2.6$ & $55 \pm 10$ & 350 \\
\hline
\end{tabular}


Contd.

\begin{tabular}{|c|c|c|c|c|c|}
\hline Star & $\mathrm{JD}(2450000+)$ & $\begin{array}{c}B_{e} \pm \sigma \\
\mathrm{G}\end{array}$ & $\begin{array}{l}V_{r} \pm \sigma \\
\mathrm{km} \mathrm{s}^{-1}\end{array}$ & $\begin{array}{c}v_{e} \sin i \pm \sigma \\
\mathrm{km} \mathrm{s}^{-1}\end{array}$ & $S / N$ \\
\hline (1) & (2) & (3) & (4) & (5) & (6) \\
\hline & 5554.240 & $\begin{array}{r}-80 \pm 70(\mathrm{r}) \\
-140 \pm 90(\mathrm{z}) \\
-30 \pm 50(\mathrm{r})\end{array}$ & $-9.0 \pm 3.1$ & & 250 \\
\hline HD 225627 & 5348.470 & $\begin{array}{l}+340 \pm 60(\mathrm{z}) \\
+320 \pm 50(\mathrm{r})\end{array}$ & $+16.3 \pm 2.8$ & $30 \pm 5$ & 300 \\
\hline & 5554.162 & $\begin{array}{l}-60 \pm 40(\mathrm{z}) \\
-20 \pm 40(\mathrm{r})\end{array}$ & $+11.1 \pm 3.1$ & & 300 \\
\hline HD 258686 & 5202.304 & $\begin{array}{l}+5750 \pm 320(\mathrm{z}) \\
+6880 \pm 450(\mathrm{~h})\end{array}$ & $+15.4 \pm 2.1$ & $35 \pm 5$ & 300 \\
\hline HD 261937 & 5554.556 & $\begin{array}{l}-720 \pm 970(\mathrm{z}) \\
+420 \pm 100(\mathrm{r})\end{array}$ & $+16.0 \pm 3.1$ & $130 \pm 10$ & 200 \\
\hline & 5555.523 & $\begin{array}{c}-1050 \pm 1460(\mathrm{z}) \\
-150 \pm 70(\mathrm{r})\end{array}$ & $+15.8 \pm 3.4$ & & 250 \\
\hline HD 279021 & 5554.458 & $\begin{array}{l}+1040 \pm 90(\mathrm{z}) \\
+860 \pm 120(\mathrm{r})\end{array}$ & $+1.3 \pm 1.2$ & $35 \pm 5$ & 250 \\
\hline & 5555.375 & $\begin{array}{l}+470 \pm 90(\mathrm{z}) \\
+490 \pm 70(\mathrm{r})\end{array}$ & $+2.9 \pm 1.2$ & & 250 \\
\hline HD 281367 & 5555.416 & $\begin{array}{c}+1250 \pm 1100(\mathrm{z}) \\
-140 \pm 100(\mathrm{r})\end{array}$ & $+46.9 \pm 3.7$ & $45 \pm 5$ & 200 \\
\hline HD 343872 & $\begin{array}{l}5281.527 \\
5282.508\end{array}$ & $\begin{array}{l}+2870 \pm 140(\mathrm{z}) \\
+1900 \pm 160(\mathrm{z}) \\
+1380 \pm 30(\mathrm{r})\end{array}$ & $-2.9 \pm 1.4$ & $25 \pm 5$ & $\begin{array}{l}200 \\
250\end{array}$ \\
\hline & 5283.524 & $0 \pm 100(\mathrm{z})$ & $-10.3 \pm 2.4$ & & 300 \\
\hline & 5284.604 & $\begin{array}{l}-820 \pm 150(\mathrm{z}) \\
-930 \pm 30(\mathrm{r})\end{array}$ & $-5.6 \pm 2.4$ & & 160 \\
\hline & 5287.552 & $\begin{array}{l}+4340 \pm 90(\mathrm{z}) \\
+4420 \pm 410(\mathrm{~h})\end{array}$ & $-3.7 \pm 2.0$ & & 200 \\
\hline & 5348.395 & $\begin{array}{l}+4280 \pm 120(\mathrm{z}) \\
+3760 \pm 40(\mathrm{r})\end{array}$ & $-4.0 \pm 2.8$ & & 200 \\
\hline$B D+53^{\circ} 1183$ & $\begin{array}{l}5202.630 \\
5311.347\end{array}$ & $\begin{array}{r}-110 \pm 120(\mathrm{z}) \\
+1030 \pm 100(\mathrm{z}) \\
-280 \pm 510(\mathrm{~h})\end{array}$ & $\begin{array}{l}+8.4 \pm 1.4 \\
+0.3 \pm 2.1\end{array}$ & $50 \pm 5$ & $\begin{array}{l}250 \\
250\end{array}$ \\
\hline
\end{tabular}


Contd.

\begin{tabular}{|c|c|c|c|c|c|}
\hline Star & JD $(2450000+)$ & $\begin{array}{c}B_{e} \pm \sigma \\
\mathrm{G}\end{array}$ & $\begin{array}{l}V_{r} \pm \sigma \\
\mathrm{km} \mathrm{s}^{-1}\end{array}$ & $\begin{array}{c}v_{e} \sin i \pm \sigma, \\
\mathrm{km} \mathrm{s}^{-1}\end{array}$ & $S / N$ \\
\hline \multirow[t]{2}{*}{ (1) } & $(2)$ & $(3)$ & (4) & $(5)$ & (6) \\
\hline & 5315.365 & $\begin{array}{l}-810 \pm 110(\mathrm{z}) \\
-510 \pm 79(\mathrm{r})\end{array}$ & $+0.9 \pm 2.8$ & & 250 \\
\hline $\mathrm{BD}+38^{\circ} 2360$ & 5555.635 & $\begin{array}{l}-50 \pm 70(\mathrm{z}) \\
+70 \pm 50(\mathrm{r})\end{array}$ & $-14.8 \pm 2.9$ & $45 \pm 5$ & 300 \\
\hline $\mathrm{BD}+37^{\circ} 431$ & 5555.169 & $\begin{array}{r}+10 \pm 30(\mathrm{z}) \\
0 \pm 40(\mathrm{r})\end{array}$ & $-10.3 \pm 1.6$ & $30 \pm 5$ & 250 \\
\hline $\mathrm{BD}+36^{\circ} 363$ & 5488.538 & $\begin{array}{r}+380 \pm 480(\mathrm{z}) \\
+50 \pm 100(\mathrm{r})\end{array}$ & $+9.8 \pm 2.9$ & $100 \pm 15$ & 250 \\
\hline \multirow[t]{2}{*}{$\mathrm{BD}+00^{\circ} 4535$} & 5406.499 & $\begin{array}{l}-570 \pm 130(\mathrm{z}) \\
-250 \pm 40(\mathrm{r})\end{array}$ & $-49.8 \pm 3.4$ & $23 \pm 5$ & 200 \\
\hline & 5553.130 & $\begin{array}{l}-1990 \pm 120(\mathrm{z}) \\
-1410 \pm 60(\mathrm{r})\end{array}$ & $-36.1 \pm 3.6$ & & 200 \\
\hline \multirow[t]{2}{*}{$\mathrm{BD}-12^{\circ} 2366$} & 5552.522 & $\begin{array}{c}-100 \pm 100(\mathrm{z}) \\
+20 \pm 40(\mathrm{r})\end{array}$ & $+32.9 \pm 2.8$ & $40 \pm 5$ & 170 \\
\hline & 5553.635 & $\begin{array}{r}+70 \pm 70(\mathrm{z}) \\
+100 \pm 80(\mathrm{r})\end{array}$ & $+37.9 \pm 2.7$ & & 170 \\
\hline NGC 752-105 & 5555.215 & $-970 \pm 620(\mathrm{z})$ & $-5.9 \pm 3.1$ & $75 \pm 10$ & 150 \\
\hline \multirow[t]{9}{*}{ o UMa* } & 5281.460 & $-30 \pm 20(\mathrm{z})$ & $+23.7 \pm 2.1$ & $23 \pm 5$ & 400 \\
\hline & 5282.294 & $\begin{array}{r}+90 \pm 20(\mathrm{z}) \\
+110 \pm 10(\mathrm{r})\end{array}$ & $+18.6 \pm 3.4$ & & 500 \\
\hline & 5283.340 & $\begin{array}{l}-110 \pm 30(\mathrm{z}) \\
-140 \pm 10(\mathrm{r})\end{array}$ & $+14.4 \pm 2.4$ & & 800 \\
\hline & 5284.336 & $\begin{array}{l}0 \pm 10(\mathrm{z}) \\
0 \pm 10(\mathrm{r})\end{array}$ & $+20.7 \pm 3.4$ & & 800 \\
\hline & 5311.311 & $-20 \pm 10(\mathrm{z})$ & $+22.0 \pm 2.8$ & & 1300 \\
\hline & 5315.328 & $-40 \pm 10(\mathrm{z})$ & $+21.2 \pm 3.7$ & & 1300 \\
\hline & 5348.268 & $\begin{array}{l}0 \pm 10(\mathrm{z}) \\
0 \pm 10(\mathrm{r})\end{array}$ & $+19.7 \pm 2.7$ & & 900 \\
\hline & 5459.470 & $-50 \pm 50(\mathrm{z})$ & $+17.0 \pm 2.8$ & & 800 \\
\hline & 5552.644 & $\begin{array}{l}-190 \pm 10(\mathrm{z}) \\
-240 \pm 20(\mathrm{r})\end{array}$ & $+15.8 \pm 1.8$ & & 1000 \\
\hline
\end{tabular}

4. COMMENTS

us earlier and have already been described in pa-

This Section gives comments and notes to the 
pers [1-3], the corresponding reference is given. More attention is given to the stars which were observed for the first time in 2010. We preserve the traditional sequence of comments adopted in the previous articles.

\subsection{Non-Magnetic Standard Stars}

We use cool slowly rotating stars with a lot of narrow lines as non-magnetic standard stars. There is no strong general magnetic field in such objects and the accuracy of field measurements is very high.

\subsubsection{HD 33256}

The star of spectral class F5. We use it as a zero standard. As it can be seen from the Table, the scattering did not exceed $50 \mathrm{G}$ in any of the cases, which fully corresponds to the expected accuracy.

$$
\text { 4.1.2. } H D 71369=o U M a
$$

A zero standard. Ten field measurements were obtained. With the exception of one night $(\mathrm{JD}=2455552.644)$, systematic errors are within $100 \mathrm{G}$. It is possible that a large deviation from zero in the given night is related to a poor adjustment of the new large CCD in the initial period of its operation.

\subsection{3. $H D 158974$}

A zero standard. In 2010, systematic measurement errors did not exceed $16 \mathrm{G}$. The details are presented in the papers dedicated to the results of 2007-2009 [1-3].

\subsubsection{HD 169191}

A zero standard. We see that in 2010 the systematic measurement errors did not exceed $20 \mathrm{G}$, except for one case (JD $=2455461.284)$ when they amounted to $142 \pm 10 \mathrm{G}$. Apparently, there have been errors of star guiding captured in the slit of the spectrograph. It could leave the slit at short exposures, and the observers did not have time to compensate for this departure.

Measurements of zero standards show that in general there are no systematic errors that could lead to a distortion of the results. Nevertheless, in some cases there are more significant deviations. Hence, if observations show that the star has a $B_{e}$ field smaller than $100 \mathrm{G}$, we do not consider the star to be magnetic even at very small formal measurement errors.

\subsection{Magnetic Standard Stars}

Chemically peculiar stars with reliably determined longitudinal field $B_{e}$ variability curves are selected as the magnetic standards. 


\subsection{1. $H D 65339=53 \mathrm{Cam}$}

One of the most well-studied magnetic CP stars. We observe it systematically for the purpose of standardization of observations [1-3]. In 2010, nine observations of this object were performed. Our results can be used when studying the long-term variability of the star.

\subsection{2. $H D 112413=\alpha^{2} C v n$}

The star with a well-known curve of the longitudinal field, the brightest of all known magnetic CP stars. It is observed for calibration. As before, the results of our measurements of the field correspond to the ephemeris. Dozens of works, devoted to the study of this star are annually published, including detailed investigations of its magnetic field.

\subsection{3. $H D 137909=\beta C r B$}

$\beta \mathrm{CrB}$ is a very well studied, second in brightness magnetic CP star. It is used to calibrate the data as the standard of the magnetic field.

\subsection{4. $H D 152107=52 \mathrm{Her}$}

52 Her is a well-studied CP star, convenient for the purposes of calibration, since the longitudinal component of the field $B_{e}$ has a constant positive polarity. In the 2010 observations a good agreement was obtained with the data of previous studies. The star is a binary, with the orbital period of about 56 years [5], therefore, we observe variability of its radial velocity.

\subsubsection{HD $201601=\gamma E q u$}

$\gamma$ Equ is a magnetic star with the longest rotation period (about 100 years). In 2010, eight measurements of the field were performed, the average value of $B_{e}$ over the year is $-1107 \pm 26 \mathrm{G}$ (by the classical Babcock method). This means that due to rotation its longitudinal field $B_{e}$ begins to depart from the phase of the negative extremum. The estimate of the field by the regression method is somewhat smaller than the value obtained in the classical way. Systematic radial velocity variations in comparison with 2009 are not noted. The average value of $V_{r}=-17.1 \pm 0.7 \mathrm{~km} \mathrm{~s}^{-1}$ within the errors coincides with the SIMBAD estimate of $V_{r}=$ $-16.5 \mathrm{~km} \mathrm{~s}^{-1}$.

\subsection{Stars with Magnetic Field Registered in} 2010

\subsubsection{HD 965}

A very slowly rotating magnetic star of the SrCrEu-type. The period of its rotation reaches 20 years. Magnetic monitoring of the star is conducted by us regularly for more than 15 years. Preliminary results are published in [6]. In 2010, the longitudinal field $B_{e}$ passed from the region of positive values to the region of negative values. The average $B_{e}$ over a year is 
-230 G. Radial velocity of the star is approximately the same as in 2009. Note that the paper for 2009 contains an annoying typo: on the date $\mathrm{JD}=2455075.438$ radial velocity is $-5.8 \mathrm{~km} \mathrm{~s}^{-1}$, instead of $+9.8 \mathrm{~km} \mathrm{~s}^{-1}$, as erroneously printed in the specified article. No signs of HD 965 binarity were ever found.

\subsection{2. $H D 5441$}

$\mathrm{SrCrEu}$ is a star of spectral class A2. The first measurements of its magnetic field were performed by us in 2009. One measurement in 2010 gave the same value of $B_{e}=-440 \pm 30 \mathrm{G}$. The lines are very narrow and sharp. The classical Zeeman and the regression method give the same field value. For more details about the star, see [3]. The GAIA parallax $\pi=2.60$ mas, the variable radial velocity of the star is $+43 \mathrm{~km} \mathrm{~s}^{-1}$ (in comparison with $+18 \mathrm{~km} \mathrm{~s}^{-1}$ and $+37 \mathrm{~km} \mathrm{~s}^{-1}$ in 2009) points to binarity. Therefore, a new magnetic binary star is discovered.

\subsection{3. $H D 5797$}

The magnetic field of this star was discovered by us earlier (see paper [7]). Semenko et al. investigated it in the work [8]. The radial velocity, measured in 2010 , was about $-3.5 \mathrm{~km} \mathrm{~s}^{-1}$, it systematically differs from that measured in 2009. We confirm that the star is binary.

\subsection{4. $H D 6757$}

A magnetic star with a strong depression in the continuum. The history of its study is described in detail in the papers [1, 2]. In [7], we reported the discovery of a field. One the discovery of a field in it. One 2010 measurement $(+2800 \mathrm{G})$ confirms the presence of a strong field, whose longitudinal component has a constant positive polarity with a weak variability relative to the mean value (about $+2700 \mathrm{G}$ ). The star is a multiple system, the main component of which has a magnetic field. It is studied in more detail in the recent work [9].

\subsubsection{HD 16705}

A chemically peculiar star with weakened helium lines [10] in the scattered NGC 1039 cluster aged $\log t=8.26$ (according to the VIZIER database).

An attempt to measure the magnetic field was performed for the first time. Owing to very wide lines with complex profiles, the $B_{e}$ value could not be reliably measured. The presence of the field can only be suspected. We estimate the projection of the rotation velocity onto the line of sight as $v_{e} \sin i=100 \mathrm{kms}^{-1}$. The VIZIER database in several quoted publications lists the rotation period of the star as $P=9.944$, but we consider it to be erroneous. Since the equatorial rotation velocity of the star is at least $100 \mathrm{~km} \mathrm{~s}^{-1}$, its period of rotation can not exceed two days. In 2010, radial velocity amounted to 
$V_{r}=-12.2 \mathrm{~km} \mathrm{~s}^{-1}$. Multiple catalogs show the presence of a faint companion at a distance of about 20". Apparently, this is an visual pair.

\subsubsection{HD 17330}

A new magnetic star. The first attempt to measure the field was carried out in 2010. The star was suspected of being magnetic, since the field was found only by the regression method, and the classical method did not allow to discover it. The measurements made in subsequent years confirmed the presence of a magnetic field in the star, the longitudinal component of which $B_{e}$ reaches $-400 \mathrm{G}$. According to the Renson and Manfroid catalog [10], the silicon lines are strengthened in the star of class B7. The SIM$\mathrm{BAD}$ database shows the radial velocity of the star of $V_{r}=-2.5 \mathrm{~km} \mathrm{~s}^{-1}$; it differs from the one we measured, $-13.6 \mathrm{~km} \mathrm{~s}^{-1}$. It is noted that the star is binary, and a companion of 11th magnitude is at a distance of $10^{\prime \prime}$. The lines in the spectrum are very narrow, the value of $v_{e} \sin i$ does not exceed $20 \mathrm{~km} \mathrm{~s}^{-1}$. Most likely, the observer sees the star at a small angle $i$.

\subsubsection{HD 29762}

A new magnetic star. A large number of narrow and sharp lines is observed in the spectrum, and hence high-accuracy measurements can be performed.

\subsubsection{HD 35298}

Borra [11] discovered this star to be magnetic. In 2010 our first Zeeman spectra of the star were obtained. Further, the observations were continued, the longitudinal field variability curve and the magnetic star model were constructed [12]. The results of measurements strongly depend on the technique used. Regression analysis gives the field two times smaller than the one yielded by the classical Babcock method. The SIMBAD database lists the radial velocity of $V_{r}=$ $+30 \mathrm{~km} \mathrm{~s}^{-1}$, which is close to the one we found. There is no data about the star's binarity.

\subsubsection{HD 35379}

A chemically peculiar star. The catalog [10] shows a SiSr peculiarity. From one 2010 measurement the presence of a magnetic field can be suspected. The star is poorly studied, other observations were not performed. There are many lines in the spectrum: $v_{e} \sin i=45 \mathrm{~km} \mathrm{~s}^{-1}$, $V_{r}=+3.7 \mathrm{~km} \mathrm{~s}^{-1}$.

\subsubsection{0. $H D 35456$}

A well-known magnetic star. Borra found its field [11]. In 2010 the field was also detected by us. Subsequently, we continued the observations of HD 35456; the results of our field measurements are published in the paper by Romanyuk et al. [13]. 


\subsubsection{HD 35881}

The star with weakened lines of helium, a member of the Orion OB1a association. Rotation is very fast: $v_{e} \sin i=200 \mathrm{~km} \mathrm{~s}^{-1}$. The first observations with a Zeeman analyzer were made in 2010. The accuracy of the field determination is very low. Subsequently, observations were continued and the results were published in Romanyuk et al. [13]. We suspected that the star is magnetic.

\subsubsection{HD 36313}

A previously known magnetic star. The field was discovered by Borra [11] using the Balmer magnetometer. However, our 2010 observation performed by the narrow metal lines did not reveal the presence of a field (see the Table). Further observations confirmed that the star is magnetic. The main component, a fast rotator possesses the field: its spectrum reveals several lines strongly broadened by rotation. Narrow lines in the spectrum belong to the secondary component, to a cooler non-magnetic star, a slow rotator. Details are described in Romanyuk et al. [13]. A member of the Orion OB1 association. Using speckle interferometry, Balega et al. [14] discovered a companion at a distance of 0 ". 15 .

\subsubsection{HD 36485}

We conducted six field measurements (two in each of the three in a row December nights in 2010). The field of negative polarity is $20 \%$ weaker when measured by the regression method in comparison with the Babcock classical method. The star with strengthened helium lines, the member of the Orion OB1b association, a spectral binary. The SIMBAD database gives the radial velocity of $V_{r}=+21 \mathrm{~km} \mathrm{~s}^{-1}$, which is close to our results. The field we found is somewhat smaller than the estimate given in the literature when measured by Landstreet's hydrogen magnetometer [15].

\subsubsection{HD 36526}

Our 2010 measurement confirmed the presence of a very strong magnetic field of the star, described in the paper [11]. Subsequently, we performed another set of measurements and determined the period of rotation of HD 36526. The results are published in Romanyuk et al. [13].

\subsubsection{HD 36540}

The star with weakened lines of helium. The magnetic field is detected, which confirmed the result of Borra [11]. The measurements were further continued. There is information indicating that the field of the star is rather weak, the longitudinal component does not exceed $1 \mathrm{kG}$. The results of a detailed study of the star were published in Romanyuk et al. [16]. 


\subsubsection{HD 36916}

The magnetic field of the star was discovered by Borra et al. [17] during a large magnetic survey of stars with weakened helium lines. We confirmed the presence of the field. Our subsequent measurements allowed to investigate the variability of the longitudinal component of the field [16].

\subsection{1\%. HD 37022}

A hot star (spectral class O7) is a member of the multiple system $\theta^{1}$ Ori C. We performed six field measurements. The value of the measured field, up to $500 \mathrm{G}$, is in agreement with the results published earlier in the literature [18]. Lines in the spectrum have a very complex profile. Field measurements are difficult. Radial velocities are variable. The star is magnetic, although not formally included in the list of peculiar stars by Renson and Manfroid [10]. A moderate linear polarization (about $0.4 \%$ ) is observed, bound, apparently, with the fact that the star is in the Great Orion Nebula.

\subsubsection{HD 37140}

The longitudinal magnetic field of the star was detected by Borra [11]. It is variable in the range from -1050 to $+400 \mathrm{G}$. Our single measurement of 2010 gives a value of $B_{e}$ of about $-500 \mathrm{G}$ by the Babcock method and $-400 \mathrm{G}$ by the regression method. Radial velocity in the SIMBAD database is $V_{r}=+14.7 \mathrm{~km} \mathrm{~s}^{-1}$, which significantly differs from the value we obtained. In the Washington Observatory catalog of binary stars HD 37140 is represented as a binary. Its companion is found at the distance of 0 ". 1 . The lines in the spectrum are narrow and sharp, which allows performing high-precision measurements of the magnetic field of the star.

\subsubsection{HD 37479}

The known magnetic star $\sigma$ OriE. In the paper [19] 22 measurements were made on a hydrogen magnetometer based on the $\mathrm{H}_{\beta}$ and He $\lambda 5876$ lines. A curve was constructed with the:

$\mathrm{JD}(\min )=2442778.819+1 \mathrm{~d} 19801$,

$B_{1}=2150 \pm 120 \mathrm{G}$,

$B_{0}=660 \pm 60 \mathrm{G}$,

$\phi_{0}=0.474$ elements. One of our 2010 measurements did not show the presence of a field, wide lines did not allow it to be measured based on metals. The measurements from the hydrogen lines are required.

\subsubsection{0. $H D 37687$}

The star with weakened lines of helium, a member of the Orion OB1c association. One observation yielded a longitudinal field of about $+500 \mathrm{G}$ in full agreement with the results of [20] obtained in 2004 and 2005. 


\subsubsection{HD 37776}

In 2010, two observations of this unique magnetic star were performed. The field measured by the Babcock method dramatically differs from that obtained by the regression method. The star was observed with the calibration aim. It is possible to see more details on this unique object in Kochukhov et al. [21]. Radial velocity within the errors coincides with that given in the SIMBAD database, what testifies in favor of the fact that the star is single.

\subsubsection{HD 38823}

The magnetic field was detected by us at the observations on the BTA [7]. The longitudinal component varies from -2500 to $+1500 \mathrm{G}$, however, the curve has not yet been constructed. The observation of 2010 was at the phase when $B_{e}$ was close to zero. The radial velocity within the error coincides with that indicated in the SIMBAD database.

\subsubsection{HD 45583}

We discovered the magnetic field of the star (see [7]), in detail the history of its magnetic research is given in the paper [3]. The longitudinal component varies with a large amplitude, the variability curve is not sinusoidal. The radial velocity reveals a weak variability. The SIMBAD database lists the value $V_{r}=+22.8 \mathrm{~km} \mathrm{~s}^{-1}$.

\subsubsection{4. $H D 49884$}

A new magnetic star. The spectral class A0, the Sr-type peculiarity. Three 2010 measurements showed the presence of a weak field, the longitudinal component of which has a negative polarity. Lines are very narrow. The radial velocity might be variable.

\subsubsection{HD 50169}

One measurement of 2010 showed that the longitudinal field of this super-slow rotator was in the phase of the period when the magnetic equator of the star is observed. The transition from the negative to positive polarity of the longitudinal field is visible. A more detailed history of studying the magnetic field of the object is described in [1]. The radial velocity $V_{r}=+14.6 \mathrm{~km} \mathrm{~s}^{-1}$ is in complete agreement with the data presented in the SIMBAD database $\left(V_{r}=+13.2 \mathrm{~km} \mathrm{~s}^{-1}\right)$.

\subsubsection{HD 50461}

The star has a large value of the photometric index $\Delta a=0.052$. We detected a magnetic field (see [7]), the results of the subsequent measurements are presented in [2]. One 2010 measurement showed the presence of a field of positive polarity. The lines in the spectrum are broadened by rotation, hence it was impossible to achieve a high accuracy of measurements. The SIMBAD database gives 
$V_{r}=+38.1 \mathrm{~km} \mathrm{~s}^{-1}$, which differs from the value we found $V_{r}=+29.5 \mathrm{~km} \mathrm{~s}^{-1}$.

\subsubsection{HD 51418}

A rare magnetic star with holmium and dysprosium anomalies [22]. Four measurements, carried out in 2010, revealed the presence of a magnetic field. The elements of photometric variability are $\mathrm{JD}(\max V)=2441241.654+5.4379$. The magnetic maximum coincides with the maximum brightness. The results of our radial velocity measurements systematically differ from those presented in the SIMBAD database: $V_{r}=-22.5 \mathrm{kms}^{-1}$. In the work of Balega et al. [14] the star revealed a companion at a distance of 0 ". 15 , weaker by three magnitudes.

\subsubsection{HD 54824}

A new magnetic star discovered by us in 2010 . All three measurements showed the presence of a magnetic field. A binary star ADS 5852AB with strengthened strontium lines. Radial velocity is variable, $v_{e} \sin i=50 \mathrm{~km} \mathrm{~s}^{-1}$.

\subsubsection{HD 89069}

A new magnetic star, the field is visible on all three spectra of 2010. According to the catalog of Renson and Manfroid [10], it has Sr$\mathrm{CrEu}$ peculiarities. The lines in the spectrum are very narrow, the accuracy of field measurements is high. The period of rotation of the star is about 18 days [23]. Speckle-interferometry allowed to resolve a companion [24] at a distance of $3 . ! 5$. Our observations showed that radial velocities are variable, but the estimates differ from $V_{r}=-10.7 \mathrm{~km} \mathrm{~s}^{-1}$ (SIMBAD).

\subsubsection{HD 96003}

A new magnetic star. One measurement of 2010 showed the presence of a weak field of negative polarity. The information about the magnetic field of the star was not previously published. Further studies confirmed the presence of a magnetic field [9] $V_{r}=-1.1 \mathrm{~km} \mathrm{~s}^{-1}$ (SIMBAD), which is quite different from ours values. The star is binary and magnetic.

\subsubsection{HD 110066}

A monitoring of the magnetic field of this very long-period star was performed. One 2010 measurement gave the value of the longitudinal magnetic field of about $-200 \mathrm{G}$. The results of previous measurements are given in Romanyuk et al. [1].

\subsubsection{2. $H D 113894$}

We have discovered a new magnetic star. All the three 2010 measurements revealed a field of positive polarity. The lines in the spectrum are very narrow, the accuracy of measurements is high. Renson and Manfroid [10] mark a $\mathrm{SrCrEu}$ peculiarity. The rotation period of the star is at 
least 10 days. The observations of the star were continued. The SIMBAD database lists the value of $V_{r}=+10 \mathrm{~km} \mathrm{~s}^{-1}$, which is quite different from what we measured. Our measurements, carried out for three consecutive nights reveal a significant scatter in radial velocity determination, but nonetheless do not go beyond the limits of formal errors. The mean out of three values we obtained is $+4.8 \pm 1.9 \mathrm{~km} \mathrm{~s}^{-1}$. There is a suspicion that the system is binary.

\subsubsection{HD 118054}

A new magnetic star discovered by us in 2010 . The field $B_{e}$ is smaller than $1 \mathrm{kG}$, of negative polarity. We determined the value of the projection of the rotation velocity onto the line of $\operatorname{sight} v_{e} \sin i=65 \mathrm{~km} \mathrm{~s}^{-1}$. According to the catalog [10], the star is of the $\mathrm{SrEuCr}$ peculiarity type. A visual binary of ADS 8954. The SIMBAD database lists $V_{r}=-14.4 \mathrm{~km} \mathrm{~s}^{-1}$, which corresponds to our measurements.

\subsubsection{HD 138633}

A new magnetic star, the field was discovered by us for the first time in 2010. The SrCrEutype peculiarity. The longitudinal component of the field is within $300 \mathrm{G}$. The chemical composition and evolutionary status were studied in the work [25]. The lines are very narrow and the accuracy the measurements are high.

\subsubsection{HD 138777}

A new magnetic star with a strong field. One 2010 measurement gave a value of $+2100 \mathrm{G}$. The lines are narrow, there are many of them. The accuracy of measurements is high. According to the catalog [10], the type of peculiarity is $\mathrm{SrEu}$. There are no data on the radial velocity in the literature. Our one measurement: $V_{r}=-46 \mathrm{~km} \mathrm{~s}^{-1}$.

\subsubsection{HD 158450}

The star with a very strong field discovered by us [7]. Four 2010 measurements showed that the extreme value of the longitudinal component of the field reaches $-4500 \mathrm{G}$. The magnetic monitoring of the star was continued. The SIMBAD database shows the radial velocity of $V_{r}=$ $-22.0 \mathrm{~km} \mathrm{~s}^{-1}$, possibly variable. In 2009, we determined the radial velocity of $-19.3 \mathrm{~km} \mathrm{~s}^{-1}$, a mean over 2010 is $V_{r}=-16.8 \mathrm{~km} \mathrm{~s}^{-1}$.

\subsubsection{HD 168856}

A peculiar star with strengthened silicon lines. Its magnetic field was found by Hubrig [26]. The longitudinal component $B_{e}=-600 \mathrm{G}$. The details can be found in the work [3]. In 2010, two measurements were taken, one of them confirmed the presence of a strong field in the star. Radial velocity $V_{r}=-9.8 \mathrm{~km} \mathrm{~s}^{-1}$, given in the SIMBAD database, differs significantly from our value of $V_{r}=-21.8 \mathrm{~km} \mathrm{~s}^{-1}$. 


\subsubsection{HD 178892}

The magnetic field of the star was found by us in 2003, a detailed article was published [27]. Since the star has the strongest field, an interest exists in regular monitoring for the purpose of searching for long-term $B_{e}$ variations. Completed in 2010, two measurements show a strong field. No variability of radial velocity throughout 2009 and 2010 wad detected. The SIMBAD database does not list any $V_{r}$ value.

\subsubsection{HD 199180}

The star with the silicon and chromium peculiarities. In the 2010 observations some signs of the field are visible. Earlier (2009 results) we discovered the field of the star. The radial velocity has the same value as and a year earlier, $V_{r}=-16.9 \mathrm{~km} \mathrm{~s}^{-1}$.

\subsubsection{HD 201174}

We started the magnetic monitoring of the star in 2006 [1]. In 2010, five observations were done. The longitudinal field has a positive sign and varies from $500 \mathrm{G}$ to $2 \mathrm{kG}$. A fairly wide scatter of radial velocities is observed.

\subsubsection{HD 225627}

A peculiar star with a large depression and strontium anomalies. Earlier (in 2009) we found the star to be magnetic. In 2010, one measurement out of two confirmed the presence of a field in the star. The lines in the spectrum are narrow. The radial velocity variability is within errors.

\subsubsection{2. $H D 258686$}

We detected the magnetic field [7]. The field in the star is very strong and reaches $+7 \mathrm{kG}$ (longitudinal component). In 2010 one measurement is done. The silicon peculiarity type. The rotation period is not found yet. ADS 5139A is a companion at a distance of $1^{\prime \prime} .5$. It is a rare case when the field from the hydrogen line core is stronger than the one determined by the classical method. A preliminary curve of $B_{e}$ variability with a period of 1.5115 days is built.

\subsubsection{HD 261937}

We first measured the magnetic field of the star in 2008 (the results are published in [2]). Two 2010 measurements give indications that the star is magnetic. For a spectral star of class $\mathrm{O} 7 \mathrm{it}$ is a fairly rare case. No data about it is listed in the catalog [10]. A binary star. The SIMBAD database lists it as a young stellar object. A member of the scattered cluster NGC 2264 aged $\log t=7.0$. Interstellar linear polarization of $0.2 \%$ is noted. 


\subsubsection{HD 279021}

The magnetic star we found [2]. The plentiful lines are narrow. The accuracy of field measurements is high. The period is $P=2$ d 80 days. Two 2010 measurements confirm the presence of the field. The star has a SrCrEu peculiarity type. No data on the radial velocity is found in the literature.

\subsubsection{5. $H D 343872$}

We discovered the star to be magnetic. The details are presented in the paper with the results of 2008 observations [2]. The field was first found in Elkin et al. [28]. Monitoring of the star continues with the goal of obtaining a magnetic curve and constructing a field model. Six measurements were made in 2008: the field strength found by the regression method is on the average $15 \%$ smaller than the one obtained by the classical method.

$$
\text { 4.3.46. } \mathrm{BD}+53^{\circ} 1183
$$

From the three 2010 measurements, we discovered a new magnetic star. The lines are narrow, the period is unknown, but it exceeds several days. Catalog [10] lists it as having strontium and chromium peculiarities. The star has been poorly studied: there is no information in the literature about radial velocity and rotation parameters. Judging by our data, radial velocity is variable, $v_{e} \sin i=50 \mathrm{~km} \mathrm{~s}^{-1}$.

$$
\text { 4.3.47. } \quad \mathrm{BD}+00^{\circ} 4535
$$

A magnetic field of $3 \mathrm{kG}$ was detected by us in 2009. Further, the presence of a strong field was confirmed: two 2010 measurements gave a field of $-0.5 \mathrm{kG}$ and $2 \mathrm{kG}$ by the classical technique and by $20 \%$ smaller than when using the regression method. Monitoring continues to obtain a phase magnetic curve.

\subsection{CP Stars without a Field Found in 2010 Observations}

\subsubsection{HD 653}

A chemically peculiar star of spectral class A0. A CrEu peculiarity type. Included in the SuperWASP list [29]. In this paper, a period of rotation of $P=1$ d 0854 days is found. The first magnetic measurements were performed by us in 2008 [2]. They did not show the presence of a field, just like the two measurements of 2010 . There are numerous lines in the spectrum, rather narrow and sharp. We found that the star is a spectroscopic binary. The lines of the second component are clearly visible in the observations made on JD $=2455488.445$; in two months the separation of components was no longer visible. The radial velocity of the main component in these two dates is very different. The projection of the rotation velocity of the main component $v_{e} \sin i$ is estimated at approximately $75 \mathrm{~km} \mathrm{~s}^{-1}$. It can be assumed that the secondary component is a slow rotator $\left(v_{e} \sin i \approx 20 \mathrm{~km} \mathrm{~s}^{-1}\right)$, it 
is fainter by approximately one magnitude. According to GAIA, the parallax is $\pi=2.93$ mas.

\subsubsection{HD 23924}

A member of the Melotte 22 cluster (the Pleiades), included into the [10] catalog, however, not indicating the peculiarity type. The spectrum of A7p. The magnetic field was not found, just like in 2007 and 2009 [1, 3]. Radial velocity is variable, the star is a spectroscopic binary. Apparently, this is an Am star. The further observations of the object are inexpedient.

\subsubsection{HD 23964}

Another member of the Melotte 22 cluster (the Pleiades). The data on it is missing in the Renson and Manfroid [10]. A spectroscopic binary. No magnetic field was found.

\subsubsection{HD 32549}

A chemically peculiar star with silicon and chromium anomalies. There exists a depression at $5200 \AA$. The 2010 measurements do not reveal the presence of a field, as well as all our previous measurements (for details, see papers $[2,3]$ ). Auriere et al. [20] performed a rather long series of observations of this star with a high spectral resolution, and in some cases the authors succeeded to register a longitudinal field of magnitude 150-200 G. Perhaps the object has a weak magnetic field. It seems that the radial velocity is variable: $V_{r}=+16.2 \mathrm{~km} \mathrm{~s}^{-1}$ in the SIMBAD; while we have obtained $+31 \mathrm{kms}^{-1}$ in 2009 and two more different values in 2010 .

\subsubsection{HD 34307}

A binary star ADS 3857AB. It is missing in the catalog of Renson and Manfroid [10]. Spectral class B9. In the observations of 2010 no magnetic field was detected.

\subsubsection{HD 34968}

A binary star. Missing in the catalog of Renson and Manfroid [10]. A fast rotator. In the 2010 observations no magnetic field was detected.

\subsubsection{HD 35101}

The star is missing in the Renson and Manfroid catalog [10]. No magnetic field was detected in two 2010 measurements, just like in 2008 [2]. The lines in the spectrum are very wide, measurements are difficult. The upper limit of the field is $500 \mathrm{G}$.

\subsubsection{HD 35548}

In the Renson and Manfroid catalog [10] it is listed as a HgMn-star. For such objects the lack of magnetic field is assumed. Our 2010 observations have not revealed the presence of the field either. A member of the Orion OB1 association. A spectroscopic binary star. 


\subsubsection{HD 35575}

The star with weakened lines of helium,a member of the Orion OB1a association. A fast rotator, the line profiles are complex, there are few of them. Accurate field measurements are difficult. In 2010, one measurement was carried out, which did not show the presence of a magnetic field. Our measurement of radial velocity, $V_{r}=+24 \mathrm{~km} \mathrm{~s}^{-1}$ significantly differs from the value given in the SIMBAD database $\left(V_{r}=+9 \mathrm{~km} \mathrm{~s}^{-1}\right)$.

\subsubsection{HD 35730}

The star with weakened lines of helium, a member of the Orion OB1a association. The spectrum has a few fairly narrow, strong, symmetrical lines. The measurements of 2010 have not revealed the field.

\subsubsection{HD 36032}

It is missing in the catalog [10]. Apparently, we observe a normal class B9 star. The reason for the star being in the list of observations is unclear. The star spatially falls into the Orion OB1 association, however, there is no evidence of its membership in it. An attempt to measure the field in the classical way revealed that this can only be done from two broad lines with complex profiles. We failed to find the field from a single spectrum.

\subsubsection{2. $H D 36629$}

A magnetic star; the field was first discovered by Borra [11]. Spectral class B3, a He-wk peculiarity [19]. The spectrum reveals very narrow and sharp lines. The star is immersed in the Parenago 1044 nebula, in whose direction a strong interstellar (or circumstellar) linear polarization is observed $(P=1.843 \%)$. According to the 2010 measurement, we have not detected any field. The longitudinal field was either not discovered later (see Fig. [30]).

\subsubsection{HD 36982}

A very hot star, a member of the youngest subgroup d of the Orion OB1 association. The age of the star is less than $1 \mathrm{Myr}$. The spectrum reveals a strong linear polarization $P=1.007 \%$. We failed to find the magnetic field. Presumably the longitudinal field of about $100 \mathrm{G}$ was found at the FORS1 VLT [18].The SIMBAD database lists the radial velocity of $V_{r}=+38 \mathrm{~km} \mathrm{~s}^{-1}$, which differs significantly from the one found by us $V_{r}=+12.6 \mathrm{~km} \mathrm{~s}^{-1}$.

\subsubsection{4. $H D 37525$}

The star with weakened lines of helium, a member of the Orion OB1b association. The brightest star in the $\sigma$ Orion cluster. The age of this cluster is estimated at 1.2 Myr. In 2010 it was observed once and magnetic field was not found. The lines are very wide $\left(v_{e} \sin i=\right.$ 
$150 \mathrm{~km} \mathrm{~s}^{-1}$ ). The measurements by the classical method are impossible to carry out, the regression method has neither yielded any field detection. In the SIMBAD database, the star is represented as a young stellar object of spectral class B5. A companion is found at a distance of 0.5 .

\subsubsection{HD 38104}

A chemically peculiar star with $\mathrm{CrEu}$ anomalies. It is described in detail in our work [2]. Since 2005, we have carried out eight observations, no magnetic field was detected. The lines are very narrow, the measurement accuracy is high, hence, based on our measurements, we estimate the upper limit of the field to be $300 \mathrm{G}$. Apparently, the radial velocity is variable, which suggests the binarity of the star.

\subsubsection{HD 39317}

Two measurements did not yield the presence of a magnetic field in this $\mathrm{SiEuCr}$ star. The results of previous years are published in Romanyuk et al. [2]. Over five years the star does not show the presence of a longitudinal field. It is unlikely that this is a very slow rotator, the magnetic field of the star is most likely below our detection limit. The radial velocity found in our work, $V_{r}=-12.1 \mathrm{~km} \mathrm{~s}^{-1}$ differs from the one given in the SIMBAD database, $V_{r}=-4.4 \mathrm{~km} \mathrm{~s}^{-1}$.

\subsubsection{HD 52711}

The magnetic field was not detected in the observations, just as before. The details can be found in Romanyuk et al. [2].

\subsubsection{HD 62512}

Two 2010 measurements did not show the presence of a field, just like earlier [2]. In the SIMBAD database $V_{r}=-4 \mathrm{~km} \mathrm{~s}^{-1}$. It means that the radial velocity is variable. Apparently, this is a binary star.

\subsubsection{HD 90763}

A chemically peculiar star with strengthened Sr lines. Three measurements performed for three consecutive nights showed that the upper limit of the longitudinal field is $B_{e}=300 \mathrm{G}$. $V_{r}=-24.8 \mathrm{~km} \mathrm{~s}^{-1}$ in the SIMBAD database. Our three values are close to the specified value. It is now too early to estimate the variability of radial velocity.

\subsubsection{HD 93294}

A poorly studied binary peculiar star. It is not represented in the Renson and Manfroid [10]. Two 2010 measurements show that if the magnetic field of the star exists, its longitudinal component does not exceed $300 \mathrm{G}$. The star is binary. 


\subsubsection{HD 97633}

HD $97633=\theta$ Leo. Measurements in December 2010 (three nights in a row) showed no field. The upper limit does not exceed 200 G. It does not make sense to examine the star for a magnetic field any longer.

\subsubsection{2. $H D 108506$}

A cool chemically peculiar star. Observations for three consecutive nights in December 2010 did not lead to the discovery of the field: the upper limit is $1 \mathrm{kG}$. The lines are wide. In the SIMBAD database it is listed as the $\delta$ Sct type. In the [10] catalog, the spectral class F1 and the $\mathrm{SrCr}$ type of peculiarity are marked. The radial velocity $V_{r}=-5.4 \mathrm{~km} \mathrm{~s}^{-1}$ (SIMBAD) does not on average differ from our 2010 measurements, but there is reason to consider it variable. The projection of the rotation velocity on the line of sight $v_{e} \sin i=150 \mathrm{~km} \mathrm{~s}^{-1}$ is similar to the one given in the VIZIER database.

\subsubsection{HD 114125}

An Algol-type eclipsing binary. In the [10] catalog, spectral class F2 and the SrEuCr type of peculiarity are listed. Two measurements of the field completed in 2010 yielded a zero result. The upper limit of the field is $200 \mathrm{G}$. The SIMBAD lists $V_{r}=-33 \mathrm{~km} \mathrm{~s}^{-1}$, which is significantly different from the value we obtained.

\subsubsection{HD 135297}

A chemically peculiar star with sharp lines. Very strong anomalies of strontium and chromium are noted in the spectrum. The photometric index is $\Delta a=0.032$. This is characteristic of magnetic stars. Two of our 2010 measurements gave a zero result. The SIMBAD gives $V_{r}=-31.9 \mathrm{~km} \mathrm{~s}^{-1}$, which significantly differs from the measurement results we have obtained. It is possible that the star is binary.

\subsubsection{5. $H D 149046$}

The star is poorly studied. In the Renson and Manfroid catalog [10] it is designated as SrCrEu-peculiar. One observation of 2010 showed the absence of a field. The radial velocity determined by us $V_{r}=-28 \mathrm{~km} \mathrm{~s}^{-1}$ is close to the one given in the SIMBAD database $\left(V_{r}=-23.5 \mathrm{~km} \mathrm{~s}^{-1}\right)$.

\subsubsection{HD 157740}

A bright CrEuSr-type star. For the search for a magnetic field was not earlier done. Our one measurement in December 2010 gave a zero result. The radial velocity is close to that given in the SIMBAD database $V_{r}=+11.2 \mathrm{~km} \mathrm{~s}^{-1}$.

\subsection{2\%. HD 178308}

A peculiar star with anomalous chromium lines. Two 2010 measurements did not lead to 
the discovery of the field. The SIMBAD database gives the value $V_{r}=-30.4 \mathrm{~km} \mathrm{~s}^{-1}$; our two measurements are in agreement with each other, but differ from the SIMBAD data, which can testify about the binarity of the star.

\subsubsection{HD 182180}

A very fast rotator, $v_{e} \sin i=300 \mathrm{~km} \mathrm{~s}^{-1}$. One 2010 measurement provides evidence of the existence of a magnetic field. Observations should be continued. Broad lines do not allow to perform accurate measurements.

\subsubsection{HD 198920}

The star with peculiarities of strontium and europium. Just like the year earlier (2009), in the reporting year no magnetic field was detected.

\subsubsection{HD217401}

A binary system ADS 16437AB. Peculiarity in Sr. We have previously found it to be magnetic (2009 results) with a longitudinal field of about -400 G. In 2010 the field was not found. Radial velocity is variable.

\subsubsection{HD 281367}

A peculiar SrEu star. The first observations were made in 2008 , they did not reveal the presence of the field. One measurement of 2010 also did not bring any evidence of the existence of the field. A fast rotator.

$$
\text { 4.4.32. } \mathrm{BD}+38^{\circ} 2360
$$

A cool peculiar SrCrEu-star. Studied poorly. The lines are narrow. The magnetic field was not detected in 2010 .

$$
\text { 4.4.33. } \mathrm{BD}+37^{\circ} 431
$$

A spectroscopic binary. An F2 spectrum. One measurement of 2010 did not reveal the presence of the field. The accuracy of the measurements is high.

$$
\text { 4.4.34. } \mathrm{BD}+36^{\circ} 363
$$

A member of the NGC 752 (RV 14) cluster. The spectral class of the star is F2p, peculiarity of Sr. One 2010 measurement did not lead to the field detection.

$$
\text { 4.4.35. } \mathrm{BD}-12^{\circ} 2366
$$

A member of the NGC 2539 cluster. It was detected as magnetic as a result of our 2008 observations. Two 2010 measurements did not show the presence of the field.

\subsubsection{NGC752-105}

The star from the NGC 752 cluster. It is not included in the catalog of Renson and Man- 
froid [10]. The field was not found in 2010, the lines in the spectrum are wide.

\section{CONCLUSION}

In 2010 we have therefore performed observations of 92 stars with a Zeeman analyzer: four of them are non-magnetic standard stars, five are standard magnetic stars with well-studied longitudinal field curves. In 47 stars the 2010 observations revealed the presence of a field, and in other 36 it was not detected. we discovered twelve new magnetic stars, and in other three stars the field is suspected.

In 2010, a large program for the study of magnetic stars in the Orion OB1 cluster was launched. Polarized spectra of over 20 objects of the association were obtained.

Most of the observed stars are poorly studied, therefore we measured radial velocities and projections of rotation velocities to the line of sight for all objects. A comparison with the literary data revealed a number of new binary stars.

\section{ACKNOWLEDGMENTS}

The authors are grateful to G. A. Chuntonov for his assistance in preparation for observations and their provision. The authors thank Russian Science Foundation for the financial support of the study (an RSF grant no. 14-50-00043).
1. I. I. Romanyuk, E. A. Semenko, and D. O. Kudryavtsev, Astrophysical Bulletin 69, 427 (2014).

2. I. I. Romanyuk, E. A. Semenko, and D.O.Kudryavtsev, Astrophysical Bulletin 70, 444 (2015).

3. I. I. Romanyuk, E. A. Semenko, D. O. Kudryavtsev, and A. V. Moiseeva, Astrophysical Bulletin 71, 302 (2016).

4. S. Bagnulo, T. Szeifert, G. A. Wade, et al., Astron. and Astrophys. 389, 191 (2002).

5. C. E. Worley and D. W. Heintz, Publ. Naval Obs. 24, 1 (1983).

6. I. I. Romanyuk, D. O. Kudryavtsev, E. A. Semenko, and I. A. Yakunin, Astrophysical Bulletin 70, 456 (2015).

7. D. O. Kudryavtsev, I. I. Romanyuk, V. G. Elkin, and E. Paunzen, Monthly Notices Royal Astron. Soc. 1372, 1804 (2006).

8. E. A. Semenko, I. A. Yakunin, and E. Yu. Kuchaeva, Astronomy Letters 37, 20 (2011).

9. E. A. Semenko, Astron. Soc. Pasific Conf. Ser. 510, 224 (2017).

10. P. Renson and J. Manfroid, Astron. and Astrophys. 498, 961 (2009).

11. E. F. Borra, Astrophys. J. 249, 398 (1981).

12. I. A. Yakunin, Astrophysical Bulletin 68, 214 (2013).

13. I. I. Romanyuk, E. A. Semenko, I. A. Yakunin, et al., Astrophysical Bulletin 71, 436 (2016).

14. Yu. Yu. Balega, V. V. Dyachenko, A. F. Maximov, et al., Astrophysical Bulletin , 67, 44 (2012). 
15. D. A. Bohlender, Astrophys. J. 346, 459 (1989).

16. I. I. Romanyuk, E. A. Semenko, I. A. Yakunin, et al., Astrophysical Bulletin 72, 165 (2017).

17. E. F. Borra, J. D. Landstreet, and I. Thompson, Astrophys. J. Suppl. 53, 151 (1983).

18. H. Grunhut, G. A. Wadem, C. Neiner, et al., Monthly Notices Royal Astron. Soc. 465, 2432 (2017).

19. D. A. Bohlender, D. N. Brown, J. D. Landstreet, and I. B. Thompson, Astrophys. J. 323, 325 (1987).

20. M. Auriere, G. A. Wade, J. Silvester, et al., Astron. and Astrophys. 475,1053 (2007).

21. O. Kochukhov, A. Lundin, I. Romanyuk, and D. Kudryavtsev, Astrophys. J. 726, 24 (2011).

22. T. J. Jones, S. C. Wolff, and W. Bonsack, Astrophys. J. 190, 579 (1974).

23. F. A. Catalano, et al., Astron. and Astrophys.
Suppl. 127, 421 (1998).

24. D. A. Rastegaev, Yu. Yu. Balega, V. V. Dyachenko, et al., Astrophysical Bulletin 69, 296 (2014).

25. A. R. Titarenko, T. A. Ryabchikova, O. P. Kochukhov, and V. V. Tsymbal, Astronomy Letters 39, 347 (2013).

26. S. Hubrig, Astronomische Nachrichten 327, 289 (2006).

27. T. A. Ryabchikova, O. P. Kochukhov, D. O. Kudryavtsev, et al., Astron. and Astrophys. 445, 47 (2006).

28. V. G. Elkin, D. O. Kudryavtsev, and I. I. Romanyuk, Astronomy Letters 28, 169 (2002).

29. E. Paunzen (private communication) (2016).

30. S. Bagnulo, L. Fossati, J. D. Landstreet, et al., Astron. and Astrophys. 583A, 115 (2015). 